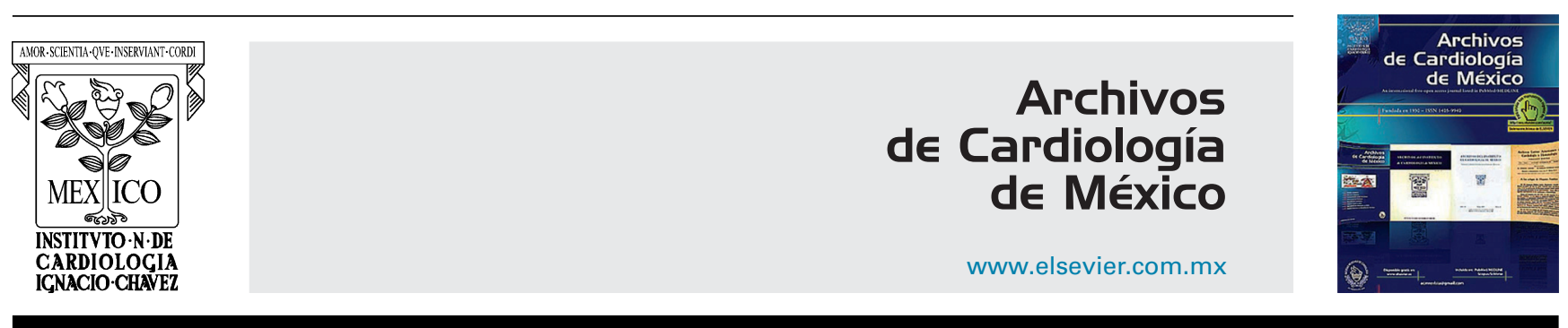

INVESTIGACIÓN CLÍNICA

\title{
Impacto de la angiografía coronaria rotacional en la cantidad total de contraste administrado y la exposición a las radiaciones ionizantes en pacientes en los que se realizan procedimientos coronarios invasivos: revisión sistemática y metaanálisis
}

\author{
Diego Fernández-Rodríguez ${ }^{\mathrm{a}, \mathrm{b}, *}$, Leydimar Anmad Shihadeh ${ }^{\mathrm{a}}$, \\ José L. Martos-Maine ${ }^{a}$, Patricia Couto-Comba ${ }^{a}$, Alejandro Quijada-Fumero ${ }^{a}$, \\ Raquel Pimienta ${ }^{a}$, José J. Grillo-Pérez ${ }^{a}$, Marcos Rodríguez-Esteban ${ }^{a}$, \\ Horacio Pérez-Hernández ${ }^{a}$, Julio Hernández-Afonso ${ }^{a}$ y Francisco Bosa Ojeda ${ }^{c}$
}

\footnotetext{
a Servicio de Cardiología, Hospital Universitario Nuestra Señora de Candelaria, Universidad de La Laguna, Santa Cruz de Tenerife, Tenerife, España

b Servicio de Cardiología, Hospital Universitari Arnau de Vilanova, Lleida, España

c Servicio de Cardiología, Hospital Universitario de Canarias, Universidad de Laguna, La Laguna, Tenerife, España
}

Recibido el 2 de agosto de 2016; aceptado el 6 de agosto de 2017

\author{
PALABRAS CLAVE \\ Angiografía \\ coronaria; \\ Angiografía coronaria \\ rotacional; \\ Angioplastia \\ coronaria; \\ Revisión sistemática; \\ Metaanálisis; \\ España
}

\begin{abstract}
Resumen
Antecedentes: Los procedimientos coronarios invasivos conllevan la administración de contraste y la exposición a radiaciones ionizantes, comportando un incremento de la morbimortalidad. La angiografía coronaria rotacional (ACR) permite adquirir múltiples proyecciones con una inyección de contraste. Hasta la fecha, no hay metaanálisis específicos comparando la $A C R$ y la angiografía coronaria convencional (ACC) en pacientes en los que se realizan procedimientos coronarios invasivos, tanto diagnósticos como diagnósticos y terapéuticos. El objetivo de este metaanálisis es evaluar el impacto de la ACR en la cantidad de contraste, y la radiación ionizante en procedimientos coronarios invasivos.

Métodos: Se realizó una búsqueda en las bases de datos PubMed y Ovid para identificar estudios tanto diagnósticos como diagnósticos y terapéuticos que comparasen ACR y ACC. Los estudios fueron evaluados sobre la calidad y los sesgos, y fueron incluidos si contemplaban alguna de las siguientes variables de valoración: volumen de contraste, radiación ionizante medida como producto dosis-área, Kerma-aire o tiempo de fluoroscopia.
\end{abstract}

* Autor para correspondencia. Hospital Universitario Nuestra Señora de Candelaria, Carretera del Rosario 145, código postal 38010, Santa Cruz de Tenerife, Tenerife, España. Fax: +922 602 334; teléfono: +651 369729.

Correo electrónico: d.fernan.2@hotmail.com (D. Fernández-Rodríguez). 
Resultados: Dieciséis estudios, totalizando 2,327 pacientes, fueron incluidos en el análisis final (1,146 pacientes recibieron ACR y 1,181, ACC), objetivándose diferencias significativas en volumen de contraste (diferencia estándar de medias [intervalo de confianza al 95\%] - 1.887 [-2.472 $a-1.302] ; p<0.001)$, producto dosis-área $(-0.726$ [-1.034 a -0.418$] ; p<0.001)$, Kerma-aire $(-0.842$ [-1.104 a -0.581$] ; p<0.001)$ y tiempo de fluoroscopia $(0.263$ [ -0.496 a -0.030$]$; $\mathrm{p}=0.027)$.

Conclusiones: La ACR permite reducir el volumen de contraste y la radiación, evaluada como producto dosis-área, Kerma-aire y tiempo de fluoroscopia en pacientes a los que se les realizan procedimientos coronarios invasivos.

(C) 2017 Instituto Nacional de Cardiología Ignacio Chávez. Publicado por Masson Doyma México S.A. Este es un artículo Open Access bajo la licencia CC BY-NC-ND (http://creativecommons. org/licenses/by-nc-nd/4.0/).

\section{KEYWORDS}

Coronary angiography; Rotational coronary angiography; Coronary angioplasty; Systematic review; Meta-analysis; Spain

\section{Introducción}

La angiografía coronaria es la técnica «gold standard» para la evaluación de las arterias coronarias, permitiendo además el tratamiento de las lesiones coronarias durante el mismo procedimiento. Sin embargo, la exposición a la radiación ionizante y al contraste yodado de los pacientes sometidos a estos procedimientos puede conllevar la aparición de efectos adversos, como disfunción renal o enfermedades oncológicas $^{1-3}$.

La realización de la angiografía coronaria mediante la técnica convencional (ACC) precisa múltiples inyecciones de contraste en diferentes proyecciones angiográficas para la adecuada evaluación de ambas arterias coronarias, necesitándose en múltiples ocasiones proyecciones adicionales para obtener una adecuada evaluación del árbol coronario $^{4,5}$.

La angiografía coronaria rotacional (ACR) simple debido a un movimiento rotacional sobre un eje del arco de fluoroscopia permite la obtención de imágenes en múltiples proyecciones angiográficas en una sola inyección de contraste, y reducir así el número de inyecciones necesarias para evaluar cada arteria coronaria ${ }^{6}$. Asimismo, una evolución de la técnica anteriormente descrita (la ACR de doble eje), al posibilitar la rotación simultánea sobre 2 ejes, permite realizar un estudio completo de cada arteria coronaria, con una única inyección de contraste para cada arteria coronaria ${ }^{7}$. De este modo, se posibilitaría una reducción en la administración de contraste y en la dosis ionizante requerida para cada estudio, y con ello los efectos indeseables asociados. 
Hasta la fecha, solo un metaanálisis ha comparado la ACR con la ACC, circunscribiéndose al escenario de la angiografía coronaria diagnóstica ${ }^{8}$. Sin embargo, los hallazgos de dicho estudio no son muy extrapolables a la práctica habitual, donde en múltiples ocasiones el diagnóstico y el tratamiento coronario se realizan durante el mismo procedimiento invasivo.

Por lo anterior, nos hemos propuesto realizar un metaanálisis comparativo de ACR y ACC, evaluando el volumen total de contraste administrado y la exposición a la radiación ionizante en pacientes sometidos a procedimientos coronarios invasivos, ya sean diagnósticos como diagnósticos y terapéuticos.

\section{Métodos}

El objetivo de este metaanálisis fue evaluar los efectos cuantitativos sobre la cantidad de contraste, y la exposición a la radiación en procedimientos coronarios invasivos, realizados en pacientes que reciben $A C R$ en comparación con la ACC. Más específicamente, se evaluó el volumen total de contraste utilizado, la exposición a la radiación medida como producto dosis-área (PDA), como Kerma-aire o como tiempo total de fluoroscopia empleado mediante la comparación de ambas estrategias.

\section{Búsqueda bibliográfica e identificación de estudios primarios}

Se realizó una revisión sistemática de la literatura publicada sobre la ACR de conformidad con la guía y los ítems específicamente reportados en el documento de consenso Preferred
Reporting Items for Systematic Reviews and Meta-Analyses (PRISMA) ${ }^{9}$.

Se realizó una búsqueda computarizada de estudios en adultos en las siguientes bases de datos electrónicas: PubMed y Ovid, sin restricción de idioma hasta el 30 de junio de 2016. Se utilizaron las siguientes palabras clave o términos: (rotational OR single axis OR dual axis) AND (coronary angiography OR coronary angioplasty OR percutaneous coronary intervention). Los estudios seleccionados debían contener información acerca de alguna de las variables de valoración a estudio, las cuales debían incluir la media y la desviación estándar, o la mediana y el rango intercuartílico, y el número de pacientes seleccionados en cada grupo. Los estudios que no cumplían estas características fueron excluidos. En caso de múltiples informes de la misma población de estudio, se utilizó la información con mayor tamaño muestral o en la que se hubiesen realizado también procedimientos coronarios terapéuticos. Dos autores (DFR y LAS) obtuvieron los artículos completos de todos aquellos que se consideraron como potencialmente relevantes para la presente revisión. Las posibles discrepancias se resolvieron mediante consenso entre ambos autores (fig. 1).

\section{Extracción de datos, evaluación de la calidad y análisis de sensibilidad}

Se reunió la siguiente información de cada uno de los artículos seleccionados: año de publicación, tipo de diseño, tamaño muestral, tipo de rotación en la angiografía (rotacional simple o rotacional de doble eje) y tipo de procedimiento realizado (diagnóstico o diagnóstico y terapéutico). Y como variables de valoración finales se utilizaron: el volumen total
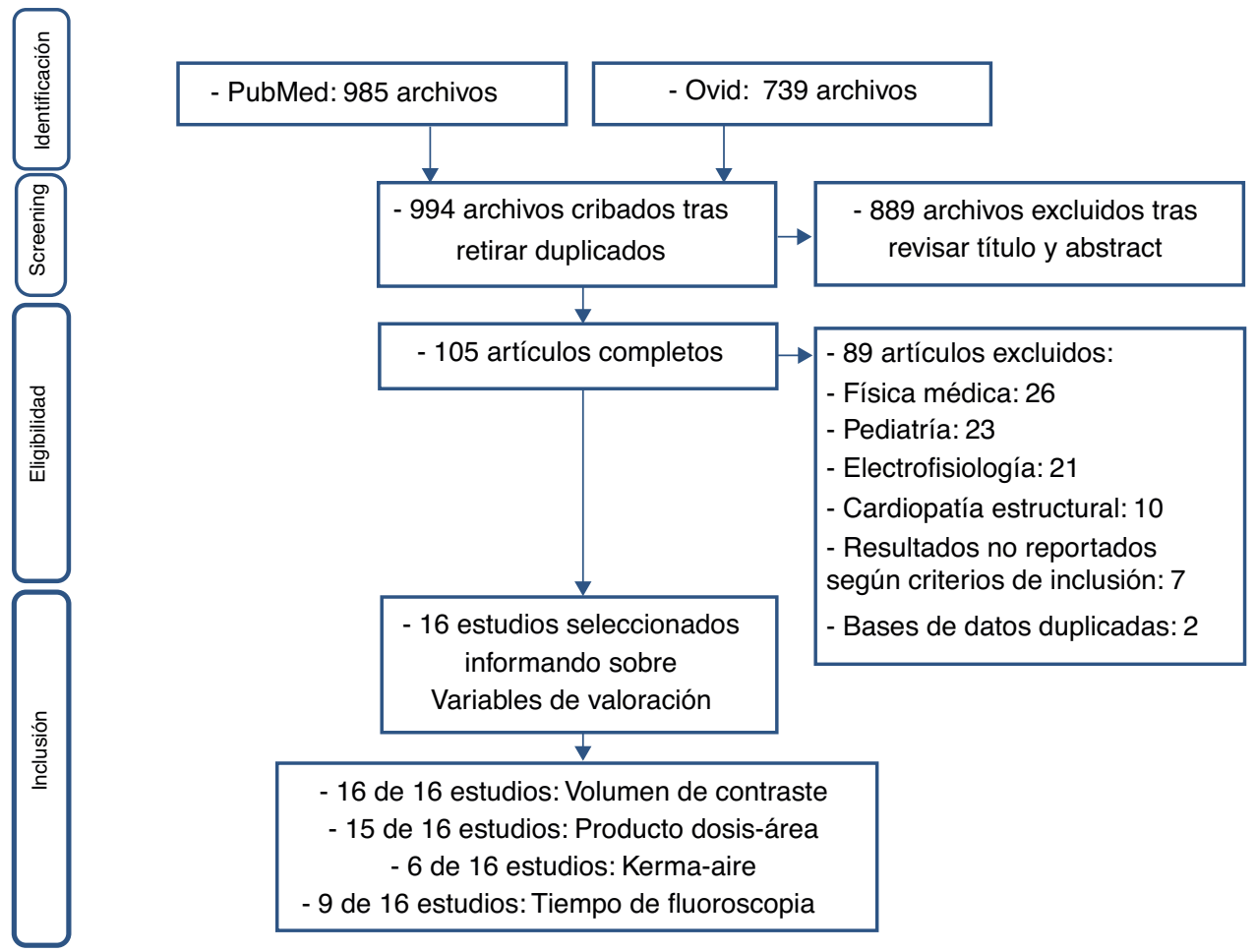

Figura 1 Diagrama de flujo que representa la búsqueda bibliográfica y la identificación de estudios primarios. 
de contraste utilizado $(\mathrm{mL})$, la radiación evaluada como PDA $\left(\mathrm{Gy} \cdot \mathrm{cm}^{2}\right)$, la radiación evaluada como Kerma-aire (mGy) y el tiempo total de fluoroscopia empleado (s). No se contactó con los autores de cada uno de los trabajos originales para ampliar la información.

Con el objeto de evaluar la calidad y los posibles sesgos de los estudios, otros 2 autores (JLMM y PCC) evaluaron los estudios seleccionados. En caso de existir discrepancias, estas se resolvieron por consenso. La calidad y los posibles sesgos fueron evaluados en los estudios aleatorizados utilizando los criterios validados de Jüni ${ }^{9}$. Para los estudios no aleatorizados se utilizó la escala de Downs modificada ${ }^{10}$. Asimismo, para las variables de valoración que combinaban más de 10 estudios originales se realizaron análisis de sensibilidad basados en el tamaño de la muestra y el tipo de procedimiento realizado (diagnóstico versus diagnóstico y terapéutico).

\section{Análisis estadístico}

Las variables continuas de las características más relevantes de los estudios originarios se presentaron como media (desviación estándar) o medianas (rango intercuartílico) según procediese. Las variables categóricas se presentaron como números absolutos. El análisis estadístico y los diagramas de bosque se realizaron utilizando el software Comprehensive Meta-Analysis (Biostat, Englewood, NJ, EE. UU.). Se consideraron como estadísticamente significativos valores de $p<0.05$. Dado que las variables de valoración finales eran continuas, los resultados se presentaron como diferencia estándar de medias con un intervalo de confianza del $95 \%$ (IC 95\%). La heterogeneidad entre estudios fue identificada utilizando el test de Chi-cuadrado y el test $\mathrm{I}^{2}$. Se utilizó un modelo de efectos fijos, en caso de no existir heterogeneidad, para comparar las variables de valoración finales. Sin embargo, en caso de detectarse heterogeneidad (Chicuadrado con valor de $\mathrm{p}$ significativo o estadístico $\mathrm{I}^{2}>50 \%$ ), se utilizó un modelo de efectos aleatorios.

\section{Resultados}

En la búsqueda bibliográfica se obtuvieron un total de 994 artículos, procedentes de las 2 bases de datos escrutadas. Después de revisar los títulos y los resúmenes de todos ellos, se seleccionaron 105 artículos a texto completo, de los cuales se excluyeron 89 por los siguientes motivos: 26 de ellos estaban relacionados con física médica, 23 artículos trataban sobre pediatría, 21 sobre electrofisiología y 10 sobre cardiopatía estructural, 7 de los artículos no reportaban los datos según los criterios de inclusión del estudio y 2 de los mismos estaban basados en datos ya utilizados en los artículos seleccionados (fig. 1). Finalmente, 16 estudios cumplieron los criterios de inclusión ${ }^{11-26}$.

Estos 16 artículos contienen datos de 2,327 pacientes. En relación con el diseño de los estudios, 9 de ellos fueron ensayos aleatorizados, en 4 de los estudios los pacientes recibieron ambos tipos de procedimiento (ACR y ACC), y 3 eran estudios observacionales (tabla 1). La calidad y los sesgos también fueron evaluados mediante herramientas específicas (tablas 2-3).
En relación con el volumen total de contraste utilizado, un total de 16 estudios ${ }^{11-26}$ fueron agrupados para el análisis, comprendiendo 1,146 pacientes en el grupo de ACR y 1,181 pacientes en el grupo de ACC. Se objetivó una diferencia significativa en cuanto al volumen total de contraste administrado favorable a la ACR (diferencia estándar de medias [IC 95\%] -1.887 [-2.472 a -1.302]; $p<0.001)$. Se empleó un modelo de efectos aleatorios debido a que existía una heterogeneidad significativa (Chi-cuadrado, $p<0.001$, test $\left.1^{2}=96.7 \%\right)$ (fig. 2).

En cuanto a la radiación evaluada mediante PDA, de 15 estudios $^{11,12,14-26}$ se extrajeron datos sobre 1,130 pacientes en el grupo de ACR y 1,166 pacientes en el grupo de ACC. Se observó una diferencia significativa entre ambos grupos a favor de la ACR (diferencia estándar de medias [IC 95\%] -0.726 [-1.034 a -0.418$] ; p<0.001)$. Para el análisis se utilizó un modelo de efectos aleatorios, dada la presencia de heterogeneidad significativa (Chi-cuadrado, $p<0.001$, test $\left.I^{2}=91.1 \%\right)$ (fig. 3).

En el análisis relativo a la radiación evaluado mediante Kerma-aire se agruparon 6 estudios ${ }^{13,15,19,22,24,26}$, suponiendo una población de 340 pacientes en el grupo de ACR y 343 pacientes en el grupo de ACC. Se detectó una diferencia estadísticamente significativa favorable a la ACR (diferencia estándar de medias [IC 95\%] -0.842 [-1.104 a -0.581]; $p<0.001)$. Es de destacar que se empleó un modelo de efectos aleatorios, dado que existía heterogeneidad significativa (Chi-cuadrado, $\mathrm{p}=0.036$, test $\mathrm{I}^{2}=58.1 \%$ ) (fig. 4).

En cuanto al tiempo total de fluoroscopia $11,13-15,21-24,26$, se agruparon un total de 9 estudios comprendiendo 608 pacientes en el grupo de ACR y 641 pacientes en el grupo de ACC. También, se observaron diferencias significativas (diferencia estándar de medias [IC 95\%]; 0.263 [-0.496 a -0.030]; $p=0.027$ ). Dada la heterogeneidad existente, se empleó un método de efectos aleatorios (Chi-cuadrado, $p=0.001$, test $\left.I^{2}=70.4 \%\right)$ (fig. 5).

\section{Discusión}

Las principales aportaciones de nuestro trabajo son las siguientes: a) el diseño de nuestra investigación permite extrapolar las conclusiones del estudio tanto a procedimientos coronarios diagnósticos como diagnósticos y terapéuticos; b) la ACR reduce la cantidad total de contraste empleado, y c) la ACR reduce la exposición a las radiaciones ionizantes.

\section{Extensión de los resultados a la práctica clínica habitual}

Los resultados generales de nuestro trabajo son congruentes con el metaanálisis previo ${ }^{8}$. Sin embargo, la principal diferencia con respecto al citado trabajo se centró en la estrategia de búsqueda y la extracción de datos de los estudios originales. La estrategia de búsqueda de nuestra investigación no contempló la exclusión de estudios en función del idioma de elaboración de los manuscritos, y además de trabajos escritos en lengua inglesa, se incorporaron trabajos escritos en otras lenguas, como el francés y el español. En cuanto a la extracción de datos, 


\begin{tabular}{|c|c|c|c|c|c|c|c|c|c|c|c|c|c|}
\hline \multirow[t]{2}{*}{ Estudio y año } & \multirow[t]{2}{*}{ Diseño } & \multicolumn{2}{|c|}{ Tipo de ACR ICP } & \multirow[t]{2}{*}{ ACR, $n$} & \multirow[t]{2}{*}{ ACC, $n$} & \multicolumn{2}{|c|}{$\begin{array}{l}\text { Volumen contraste } \\
(\mathrm{mL}) \text {, media } \\
(\mathrm{DE}) / \text { mediana }(\mathrm{RIC})\end{array}$} & \multicolumn{2}{|c|}{$\begin{array}{l}\text { PDA }\left(\mathrm{Gy} \cdot \mathrm{cm}^{2}\right) \text {, } \\
\text { media } \\
(\mathrm{DE}) / \text { mediana } \\
(\mathrm{RIC})\end{array}$} & \multicolumn{2}{|c|}{$\begin{array}{l}\text { Kerma-aire (mGy), } \\
\text { media (DE)/mediana } \\
\text { (RIC) }\end{array}$} & \multicolumn{2}{|c|}{$\begin{array}{l}\text { Fluoroscopia (s), media } \\
\text { (DE)/mediana (RIC) }\end{array}$} \\
\hline & & & & & & ACR & ACC & ACR & $\mathrm{ACC}$ & ACR & $\mathrm{ACC}$ & ACR & ACC \\
\hline $\begin{array}{l}\text { Kuon et al. }{ }^{11}, \\
2002\end{array}$ & $\begin{array}{l}\text { ACR y ACC en } \\
\text { cada paciente }\end{array}$ & Simple & No & 15 & 15 & $25(4)$ & $64(9)$ & $9(4)$ & $7(3)$ & - & - & $126(74)$ & $156(79)$ \\
\hline $\begin{array}{l}\text { Maddux et al. }{ }^{12}, \\
2004\end{array}$ & Aleatorizado & Simple & No & 28 & 28 & $36(13)$ & $53(11)$ & $39(19)$ & $54(23)$ & - & - & - & - \\
\hline $\begin{array}{l}\text { Rigattieri } \\
\quad \text { et al. }{ }^{13}, 2005\end{array}$ & Aleatorizado & Simple & No & 16 & 15 & $77(22)$ & $103(26)$ & - & - & $531(272)$ & $831(344)$ & $234(90)$ & $294(108)$ \\
\hline $\begin{array}{l}\text { Akhtar et al. }{ }^{14} \text {, } \\
\quad 2005\end{array}$ & Aleatorizado & Simple & No & 25 & 25 & $24(5)$ & $40(10)$ & $35(14)$ & $30(20)$ & - & - & $44(33)$ & $44(40)$ \\
\hline $\begin{array}{l}\text { Smida et al. }{ }^{15} \text {, } \\
2007\end{array}$ & Aleatorizado & Simple & No & 32 & 33 & $60(10)$ & $90(15)$ & $18(9)$ & $24(11)$ & 449 (228) & $700(384)$ & $282(168)$ & $330(246)$ \\
\hline $\begin{array}{l}\text { Garcia et al. }{ }^{16}, \\
\quad 2009\end{array}$ & $\begin{array}{l}\text { ACR y ACC en } \\
\text { cada paciente }\end{array}$ & Simple & No & 100 & 100 & $24(3)$ & $39(4)$ & $27(4)$ & $32(4)$ & - & - & - & - \\
\hline $\begin{array}{l}\text { Empen et al. }{ }^{17}, \\
2010\end{array}$ & $\begin{array}{l}\text { ACR y ACC en } \\
\text { cada paciente }\end{array}$ & Simple & No & 207 & 207 & $32(5)$ & $52(8)$ & $5(3)$ & $12(6)$ & - & - & - & - \\
\hline $\begin{array}{l}\text { Klein et al. }{ }^{18} \text {, } \\
2011\end{array}$ & $\begin{array}{l}\text { ACR y ACC en } \\
\text { cada paciente }\end{array}$ & Doble & No & 21 & 21 & $27(5)$ & $53(12)$ & $24(6)$ & $38(12)$ & $1,278(865)$ & $1,860(1,474)$ & - & - \\
\hline $\begin{array}{l}\text { Gómez-Menchero } \\
\text { et al. }{ }^{19}, 2012\end{array}$ & Aleatorizado & Doble & Sí & 28 & 29 & 239 (114) & 335 (192) & $90(64)$ & $110(91)$ & - & - & - & - \\
\hline Liu et al. $.^{20}, 2012$ & Aleatorizado & Doble & No & 94 & 95 & $30(6)$ & $52(10)$ & $10(4)$ & $21(8)$ & - & - & - & - \\
\hline $\begin{array}{l}\text { Grech et al. }{ }^{21}, \\
2012\end{array}$ & Aleatorizado & Doble & No & 216 & 247 & $24(8)$ & $51(19)$ & 27 (17) & $44(23)$ & - & - & $115(80)$ & $168(168)$ \\
\hline $\begin{array}{l}\text { Yasar et al. }{ }^{22} \text {, } \\
2013\end{array}$ & Observacional & Doble & No & 56 & 61 & $54(27)$ & $71(25)$ & $34(24)$ & $67(49)$ & 385 (235) & 770 (469) & $366(204)$ & $378(282)$ \\
\hline $\begin{array}{l}\text { Eloot et al. }{ }^{23}, \\
2013\end{array}$ & Aleatorizado & Simple & No & 40 & 40 & $73(41-111)$ & $104(67-190)$ & $33(12-94)$ & $50(19-177)$ & - & - & $110(35-492)$ & $150(43-833)$ \\
\hline $\begin{array}{l}\text { Giuberti et al. }{ }^{24} \text {, } \\
2014\end{array}$ & Aleatorizado & Doble & No & 101 & 100 & $60(53-72)$ & $76(68-87)$ & $20(13-29)$ & $30(21-37)$ & $270(176-451)$ & $542(371-721)$ & $181(150-229)$ & $165(132-214)$ \\
\hline $\begin{array}{l}\text { Farshid et al. }{ }^{25}, \\
\quad 2014\end{array}$ & Observacional & Doble & No & 107 & 105 & $26(8)$ & $42(12)$ & $16(11)$ & $30(19)$ & 189 (127) & $430(261)$ & $147(96)$ & $200(136)$ \\
\hline $\begin{array}{l}\text { Morris et al. }{ }^{26}, \\
2016\end{array}$ & Observacional & Simple & Sí & 60 & 60 & $342(188)$ & 311 (187) & $60(40)$ & $48(35)$ & - & - & - & - \\
\hline
\end{tabular}

ACC: angiografía coronaria convencional; ACR: angiografía coronaria rotacional; DE: desviación estándar; ICP: intervencionisno coronario percutáneo; PDA: producto dosis-área; RIC: riesgo intercuartílico;

Los datos numéricos que presentaban decimales en los estudios originales fueron redondeados a la unidad más próxima para facilitar la comprensión de la tabla. 
Tabla 2 Evaluación de la calidad y los sesgos para los estudios aleatorizados (criterios de Jüni)

\begin{tabular}{|c|c|c|c|c|c|c|c|c|c|}
\hline Estudios & $\begin{array}{l}\text { Maddux } \\
\text { et al. }{ }^{12} \text {, } \\
2004\end{array}$ & $\begin{array}{l}\text { Rigattieri } \\
\text { et al. }{ }^{13} \\
2005\end{array}$ & $\begin{array}{l}\text { Akhtar } \\
\text { et al. }{ }^{14} \\
2005\end{array}$ & $\begin{array}{l}\text { Smida } \\
\text { et al. }{ }^{15} \\
2007\end{array}$ & $\begin{array}{l}\text { Gómez- } \\
\text { Menchero } \\
\text { et al. }{ }^{19} \\
2012\end{array}$ & $\begin{array}{l}\text { Liu } \\
\text { et al. }{ }^{20} \text {, } \\
2012\end{array}$ & $\begin{array}{l}\text { Grech } \\
\text { et al. }{ }^{21} \\
2012\end{array}$ & $\begin{array}{l}\text { Eloot } \\
\text { et al. }{ }^{23} \\
2013\end{array}$ & $\begin{array}{l}\text { Giuberti } \\
\text { et al. }{ }^{24} \text {, } \\
2014\end{array}$ \\
\hline \multicolumn{10}{|l|}{ Sesgo de selección } \\
\hline $\begin{array}{l}\text { 1. ¿Fue la asignación } \\
\text { adecuada? Centralizado, } \\
\text { código numérico, sobres } \\
\text { opacos }\end{array}$ & $\mathrm{D}$ & $\mathrm{D}$ & $\mathrm{D}$ & $\mathrm{D}$ & $\mathrm{D}$ & $\mathrm{D}$ & $\mathrm{D}$ & $\mathrm{D}$ & $\mathrm{D}$ \\
\hline $\begin{array}{l}\text { 2. ¿Fue el método de } \\
\text { aleatorización } \\
\text { adecuadamente } \\
\text { descrito? }\end{array}$ & $\mathrm{N}$ & $\mathrm{N}$ & $\mathrm{S}$ & $\mathrm{N}$ & $\mathrm{S}$ & $\mathrm{S}$ & $\mathrm{S}$ & $\mathrm{N}$ & $\mathrm{S}$ \\
\hline $\begin{array}{l}\text { 3. ¿Fueron los grupos } \\
\text { similares al inicio del } \\
\text { estudio? }\end{array}$ & $\mathrm{S}$ & $S$ & $\mathrm{~S}$ & $\mathrm{~S}$ & $\mathrm{~S}$ & $\mathrm{~S}$ & $\mathrm{~S}$ & $S$ & $\mathrm{~S}$ \\
\hline \multicolumn{10}{|l|}{ Sesgo de realización } \\
\hline $\begin{array}{l}\text { 4. ¿Fueron los } \\
\text { pacientes/cuidadores } \\
\text { cegados respecto a la } \\
\text { intervención? }\end{array}$ & $N$ & $\mathrm{~N}$ & $N$ & $N$ & $N$ & $N$ & $N$ & $\mathrm{~N}$ & $\mathrm{~N}$ \\
\hline \multicolumn{10}{|l|}{ Sesgo de selección } \\
\hline $\begin{array}{l}5 . \text { ¿Se comprobaron los } \\
\text { resultados de forma } \\
\text { ciega? }\end{array}$ & $\mathrm{S}$ & $S$ & $\mathrm{~S}$ & $\mathrm{~S}$ & $\mathrm{~S}$ & $\mathrm{~S}$ & $\mathrm{~S}$ & $S$ & $\mathrm{~S}$ \\
\hline \multicolumn{10}{|l|}{ Sesgo de deserción } \\
\hline $\begin{array}{l}\text { 6. ¿Cuál fue el } \\
\text { porcentaje de pérdidas } \\
\text { en el estudio? }\end{array}$ & 0 & 0 & 0 & 0 & 0 & 0 & 0 & 0 & 0 \\
\hline $\begin{array}{l}\text { 7. ¿Fueron todos los } \\
\text { pacientes analizados en } \\
\text { el grupo al que fueron } \\
\text { asignados (análisis por } \\
\text { intención de tratar)? }\end{array}$ & $\mathrm{S}$ & $S$ & $\mathrm{~S}$ & $\mathrm{~S}$ & $\mathrm{~S}$ & $\mathrm{~S}$ & $\mathrm{~S}$ & $\mathrm{~S}$ & $\mathrm{~S}$ \\
\hline
\end{tabular}

D: desconocido; N: no; S: sí.

no solo se utilizaron estudios que contemplasen procedimientos diagnósticos, sino también terapéuticos. Todo ello posibilitó incluir finalmente 16 estudios en el análisis combinado, y así aumentar el número total de pacientes de nuestro estudio. Por estos motivos, consideramos que nuestro trabajo se adecua en mayor medida a la práctica habitual, en la que se suelen realizar los procedimientos coronarios diagnósticos y terapéuticos en un único acto médico.

\section{Reducción en la administración de contraste y en la exposición a las radiaciones ionizantes}

Una de las principales complicaciones asociadas a los procedimientos coronarios invasivos es la aparición de nefropatía inducida por contraste. Dicha condición puede alcanzar a un tercio de los pacientes derivados para procedimientos coronarios invasivos, siendo una de las causas más comunes de insuficiencia renal adquirida en los pacientes cardiológicos ${ }^{1,2}$. El desarrollo de dicha complicación se asocia con una hospitalización prolongada, un destacado incremento en la morbilidad, así como en la mortalidad a corto y largo plazo, y un aumento en los costos sanitarios $^{1,2,27}$. Debido a que el volumen de contraste empleado en los procedimientos angiográficos se relaciona estrechamente con la aparición de nefropatía inducida por contraste $^{28}$, la implementación de técnicas como la ACR, que reducen la administración de contraste, podría posibilitar la disminución de la incidencia de esta peligrosa complicación.

En relación con la exposición a las radiaciones ionizantes, es reseñable que en los últimos tiempos se ha producido un gran incremento en la realización de procedimientos angiográficos cardiacos y vasculares periféricos ${ }^{29,30}$. Debido a este gran incremento en el número de procedimientos, se hace patente la necesidad de implementar técnicas que permitan limitar la exposición de los pacientes y del personal médico a las radiaciones ionizantes, y a los riesgos que conllevan, principalmente derivados de procesos oncológicos ${ }^{31}$. Nuestros resultados muestran una reducción significativa en la radiación que recibe el paciente (PDA), así como en la 
Tabla 3 Evaluación de la calidad y los sesgos para los estudios aleatorizados (escala de Downs modificada)

\begin{tabular}{|c|c|c|c|c|c|c|c|}
\hline Estudios & $\begin{array}{l}\text { Kuon } \\
\text { et al. }{ }^{11} \\
2002\end{array}$ & $\begin{array}{l}\text { García } \\
\text { et al. }{ }^{16} \\
2009\end{array}$ & $\begin{array}{l}\text { Empen } \\
\text { et al. }{ }^{17} \\
2010\end{array}$ & $\begin{array}{l}\text { Klein } \\
\text { et al. } \\
2011\end{array}$ & $\begin{array}{l}\text { Yasar } \\
\text { et al.22, } \\
2013\end{array}$ & $\begin{array}{l}\text { Farshid } \\
\text { et al. } \\
2014\end{array}$ & $\begin{array}{l}\text { Morris } \\
\text { et al. }{ }^{26} \text {, } \\
2016\end{array}$ \\
\hline \multicolumn{8}{|l|}{ Escala modificada de Downs } \\
\hline \multicolumn{8}{|l|}{ Descripción de la información } \\
\hline 1. Hipótesis/objetivo & $\mathrm{S}$ & $\mathrm{S}$ & $\mathrm{S}$ & $\mathrm{S}$ & $\mathrm{S}$ & $\mathrm{S}$ & $\mathrm{S}$ \\
\hline 2. Resultados principales & $\mathrm{S}$ & $\mathrm{S}$ & $\mathrm{S}$ & $\mathrm{S}$ & $\mathrm{S}$ & $\mathrm{S}$ & $\mathrm{S}$ \\
\hline 3. Criterios de inclusión/exclusión & $S$ & $\mathrm{~S}$ & $\mathrm{~S}$ & $\mathrm{~S}$ & $\mathrm{~S}$ & $\mathrm{~S}$ & $\mathrm{~S}$ \\
\hline 4. Intervención de interés & $\mathrm{S}$ & $S$ & $\mathrm{~S}$ & $\mathrm{~S}$ & $\mathrm{~S}$ & $\mathrm{~S}$ & $\mathrm{~S}$ \\
\hline $\begin{array}{l}\text { 5. Distribución de factores de confusión entre } \\
\text { grupos }\end{array}$ & $\mathrm{N}$ & $\mathrm{S}$ & $\mathrm{S}$ & $\mathrm{S}$ & $\mathrm{S}$ & $\mathrm{S}$ & $\mathrm{S}$ \\
\hline 6. Descripción del hallazgo principal & $S$ & $\mathrm{~S}$ & $\mathrm{~S}$ & $\mathrm{~S}$ & $\mathrm{~S}$ & $\mathrm{~S}$ & $\mathrm{~S}$ \\
\hline $\begin{array}{l}\text { 7. Rango intercuartílico o desviación estándar o } \\
\text { error estándar de la media }\end{array}$ & $\mathrm{S}$ & $\mathrm{S}$ & $\mathrm{S}$ & $\mathrm{S}$ & $\mathrm{S}$ & $\mathrm{S}$ & $\mathrm{S}$ \\
\hline 8. Eventos adversos & $\mathrm{N}$ & $\mathrm{N}$ & $\mathrm{S}$ & $\mathrm{S}$ & $\mathrm{N}$ & $\mathrm{S}$ & $\mathrm{N}$ \\
\hline \multicolumn{2}{|l|}{ 9. Características de las pérdidas en el seguimientoN/A } & $\mathrm{N} / \mathrm{A}$ & $\mathrm{N} / \mathrm{A}$ & $\mathrm{N} / \mathrm{A}$ & $\mathrm{N} / \mathrm{A}$ & $\mathrm{N} / \mathrm{A}$ & N/A \\
\hline 10. Valor exacto de $p(0<0.001)$ & $\mathrm{S}$ & $\mathrm{S}$ & $\mathrm{S}$ & $\mathrm{S}$ & $\mathrm{S}$ & $\mathrm{S}$ & $\mathrm{S}$ \\
\hline \multicolumn{8}{|l|}{ Validez externa } \\
\hline 11. Se solicitó la participación & $\mathrm{S}$ & $\mathrm{S}$ & $\mathrm{S}$ & $\mathrm{S}$ & $\mathrm{N}$ & $\mathrm{S}$ & $\mathrm{N}$ \\
\hline 12. Representatividad de la población general & $\mathrm{S}$ & $\mathrm{S}$ & $\mathrm{S}$ & $\mathrm{S}$ & $\mathrm{S}$ & $\mathrm{S}$ & $\mathrm{S}$ \\
\hline 13. Equipo de trabajo, lugar, recursos adecuados & $\mathrm{S}$ & $\mathrm{S}$ & $\mathrm{S}$ & $\mathrm{S}$ & $\mathrm{S}$ & $\mathrm{S}$ & $\mathrm{S}$ \\
\hline \multicolumn{8}{|l|}{ Validez interna-sesgos } \\
\hline 14. Cegamiento de los pacientes & $\mathrm{N}$ & $\mathrm{N}$ & $\mathrm{N}$ & $\mathrm{N}$ & $\mathrm{N}$ & $\mathrm{N}$ & $\mathrm{N}$ \\
\hline 15. Cegamiento de los evaluadores & $\mathrm{N}$ & $\mathrm{N}$ & $\mathrm{N}$ & $\mathrm{N}$ & $\mathrm{N}$ & $\mathrm{N}$ & $\mathrm{N}$ \\
\hline 16. Se especificaron análisis post-hoc & $\mathrm{N}$ & $\mathrm{N}$ & $\mathrm{N}$ & $\mathrm{N}$ & $\mathrm{N}$ & $\mathrm{N}$ & $\mathrm{N}$ \\
\hline 17. Ajuste por la duración del seguimiento & $\mathrm{N} / \mathrm{A}$ & N/A & $\mathrm{N} / \mathrm{A}$ & $\mathrm{N} / \mathrm{A}$ & $\mathrm{N} / \mathrm{A}$ & $\mathrm{N} / \mathrm{A}$ & N/A \\
\hline 18. Complianza & $\mathrm{S}$ & $\mathrm{S}$ & $\mathrm{S}$ & $\mathrm{S}$ & $\mathrm{S}$ & $\mathrm{S}$ & $\mathrm{S}$ \\
\hline 19. Principal resultado representativo o apropiado & $\mathrm{S}$ & $\mathrm{S}$ & $\mathrm{S}$ & $\mathrm{S}$ & $\mathrm{S}$ & $\mathrm{S}$ & $\mathrm{S}$ \\
\hline \multicolumn{8}{|l|}{ Validez interna-factores de confusión } \\
\hline 20. Misma población & $\mathrm{S}$ & $\mathrm{S}$ & $\mathrm{S}$ & $\mathrm{S}$ & $\mathrm{S}$ & $\mathrm{S}$ & $\mathrm{S}$ \\
\hline 21. Mismo lapso temporal & $\mathrm{S}$ & $\mathrm{S}$ & $\mathrm{S}$ & $\mathrm{S}$ & $\mathrm{S}$ & $\mathrm{S}$ & $\mathrm{S}$ \\
\hline 22. Análisis ajustados sobre el resultado principal & $\mathrm{S}$ & $\mathrm{S}$ & $\mathrm{S}$ & $\mathrm{S}$ & $\mathrm{S}$ & $\mathrm{S}$ & $\mathrm{S}$ \\
\hline 23. Pérdidas en el seguimiento valoradas & N/A & N/A & $\mathrm{N} / \mathrm{A}$ & N/A & N/A & N/A & N/A \\
\hline \multicolumn{8}{|l|}{ Poder } \\
\hline $\begin{array}{l}\text { 24. ¿Tiene el estudio suficiente poder para } \\
\text { detectar un efecto clínico importante donde el valor } \\
\text { de probabilidad para encontrar una diferencia debida } \\
\text { al azar sea inferior al } 5 \% \text { ? }\end{array}$ & S & $\mathrm{S}$ & $\mathrm{S}$ & $\mathrm{S}$ & $\mathrm{S}$ & $\mathrm{S}$ & $\mathrm{S}$ \\
\hline
\end{tabular}

N/A: no aplicable (no se realizó seguimiento de los pacientes, dado que las variables de valoración eran angiográficas; por ejemplo, volumen de contraste, producto dosis-área, etc.); N: no; S: sí.

radiación dispersa (Kerma-aire) y el tiempo de fluoroscopia, y también están en línea con los de Loomba et al. ${ }^{8}$. Asimismo, también debemos señalar que aunque las dosis de radiaciones ionizantes son menores en los procedimientos diagnósticos que en los terapéuticos, pueden suponer más de un tercio de la dosis acumulada en los procedimientos combinados (diagnósticos y terapéuticos) ${ }^{32,33}$. Por lo tanto, la generalización de la ACR podría contribuir a una mayor reducción de la exposición a las radiaciones ionizantes tanto de los procedimientos simples como de los complejos.

\section{Limitaciones}

La limitación más destacable de nuestro trabajo es la heterogeneidad presente en las variables de valoración. Por este motivo, en dichos casos se emplearon modelos de efectos aleatorios. No obstante, los resultados de las variables de valoración que comprenden más de 10 estudios (volumen de contraste y PDA) permanecieron similares tras realizar el análisis de sensibilidad, que excluía los estudios de menor tamaño de la muestra y aquellos que incluían procedimientos diagnósticos y terapéuticos. 


\section{Contraste}

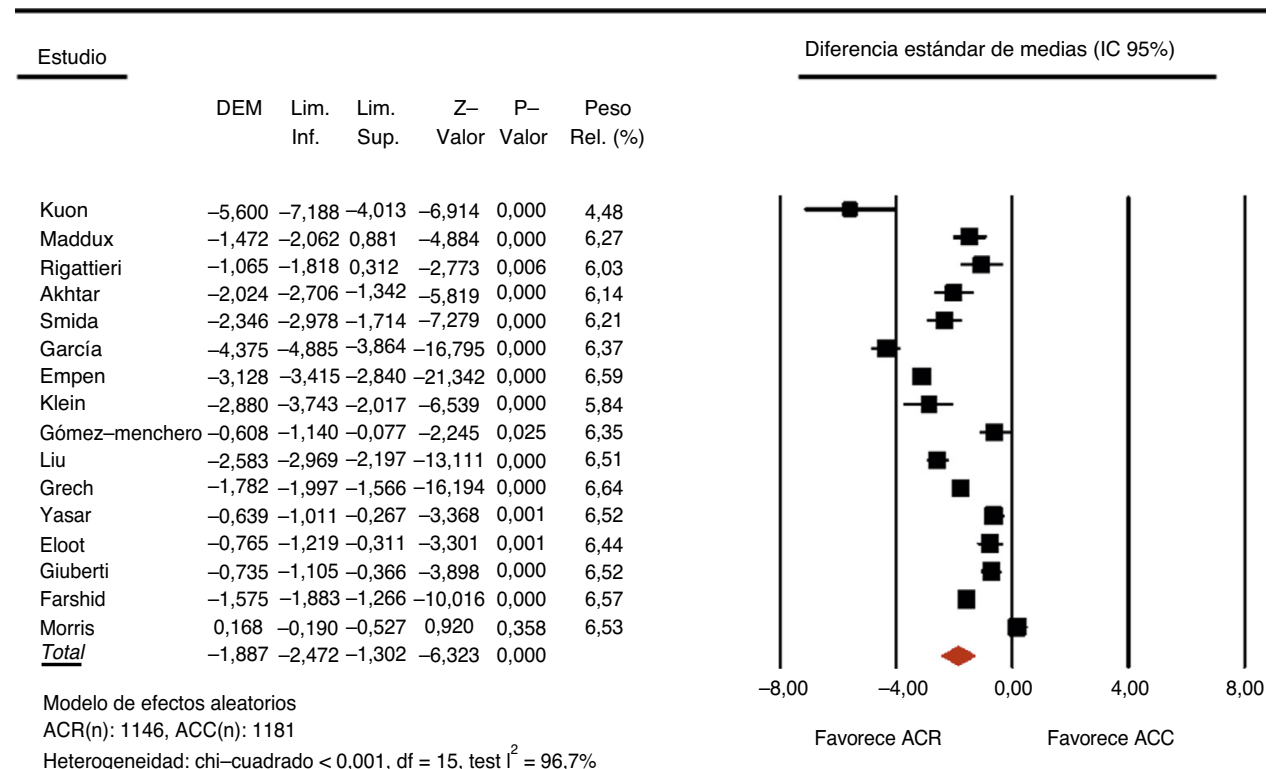

Figura 2 Diagrama de bosque que compara el volumen de contraste administrado $(\mathrm{mL})$ entre la angiografía coronaria rotacional (ACR) y la angiografía coronaria convencional (ACC). DEM: diferencia estándar de medias; IC 95\%: intervalo de confianza del 95\%; Inf.: inferior; Lim.: límite; Rel.: relativo; Sup.: superior.

Producto dosis-área

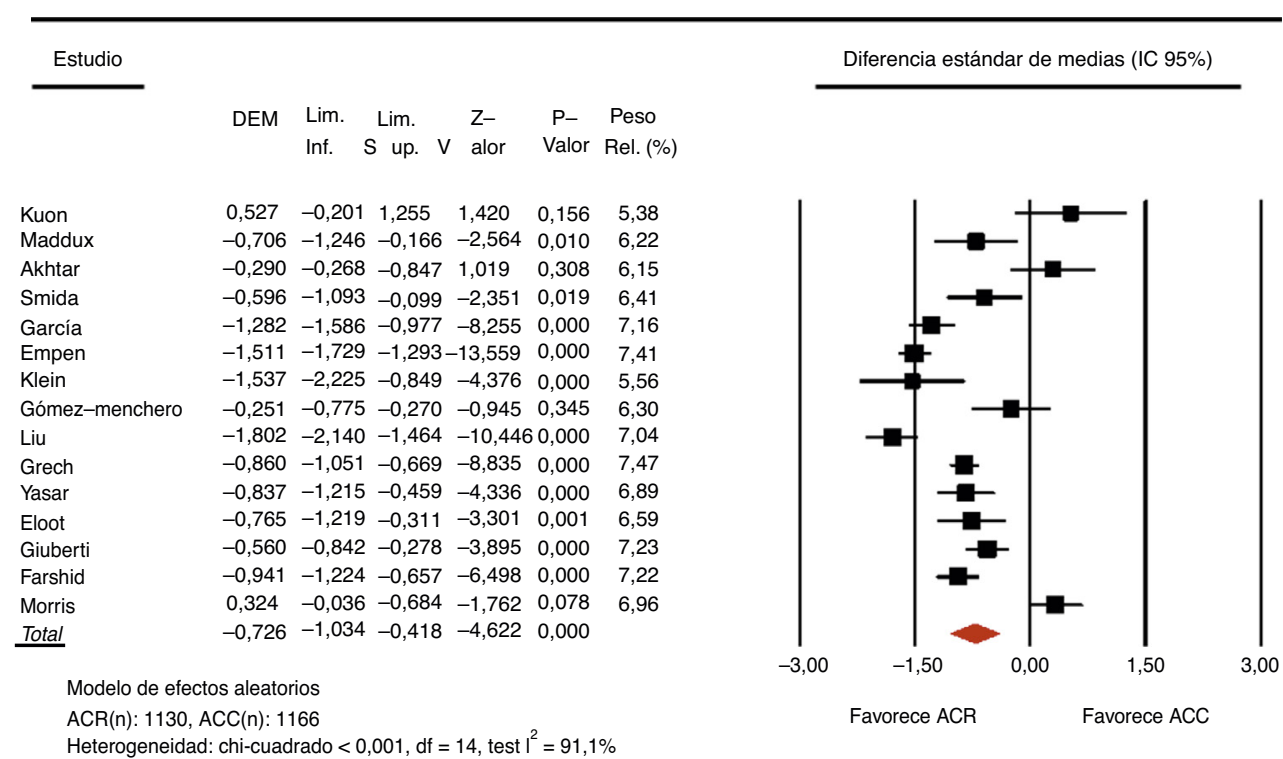

Figura 3 Diagrama de bosque que compara la radiación evaluada mediante producto dosis-área $\left(\mathrm{Gy} \cdot \mathrm{cm}^{2}\right)$ entre la angiografía coronaria rotacional (ACR) y la angiografía coronaria convencional (ACC). DEM: diferencia estándar de medias; IC 95\%: intervalo de confianza del 95\%; Inf.: inferior; Lim.: límite; Rel.: relativo; Sup.: superior. 


\section{Kerma-aire}

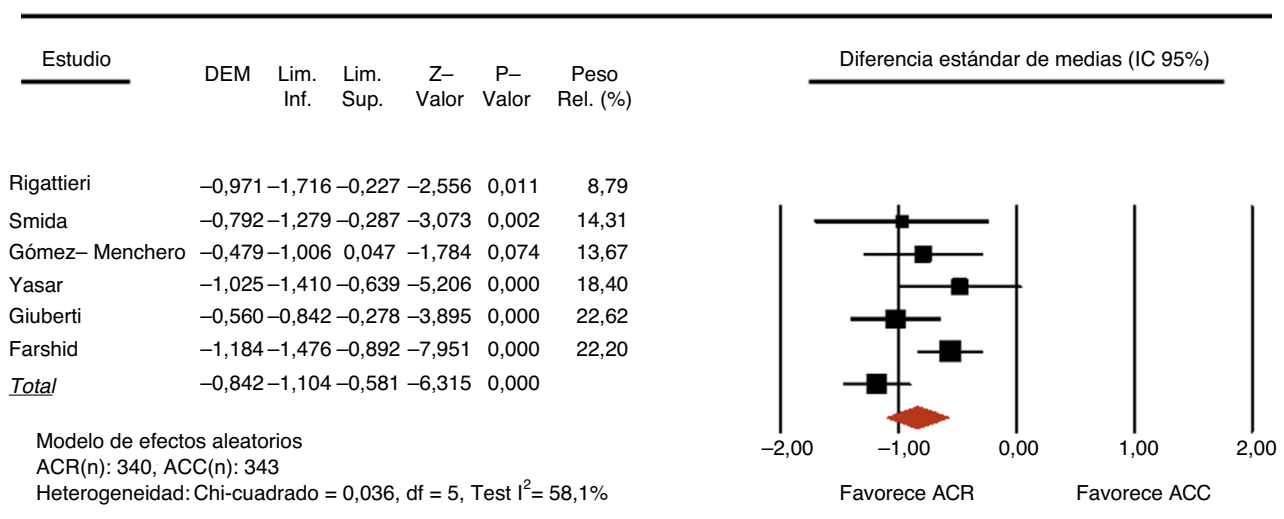

Figura 4 Diagrama de bosque que compara la radiación evaluada mediante Kerma-aire (mGy) entre la angiografía coronaria rotacional (ACR) y la angiografía coronaria convencional (ACC). DEM: diferencia estándar de medias; IC 95\%: intervalo de confianza del 95\%; Inf.: inferior; Lim.: límite; Rel.: relativo; Sup.: superior.

Tiempo de fluoroscopia

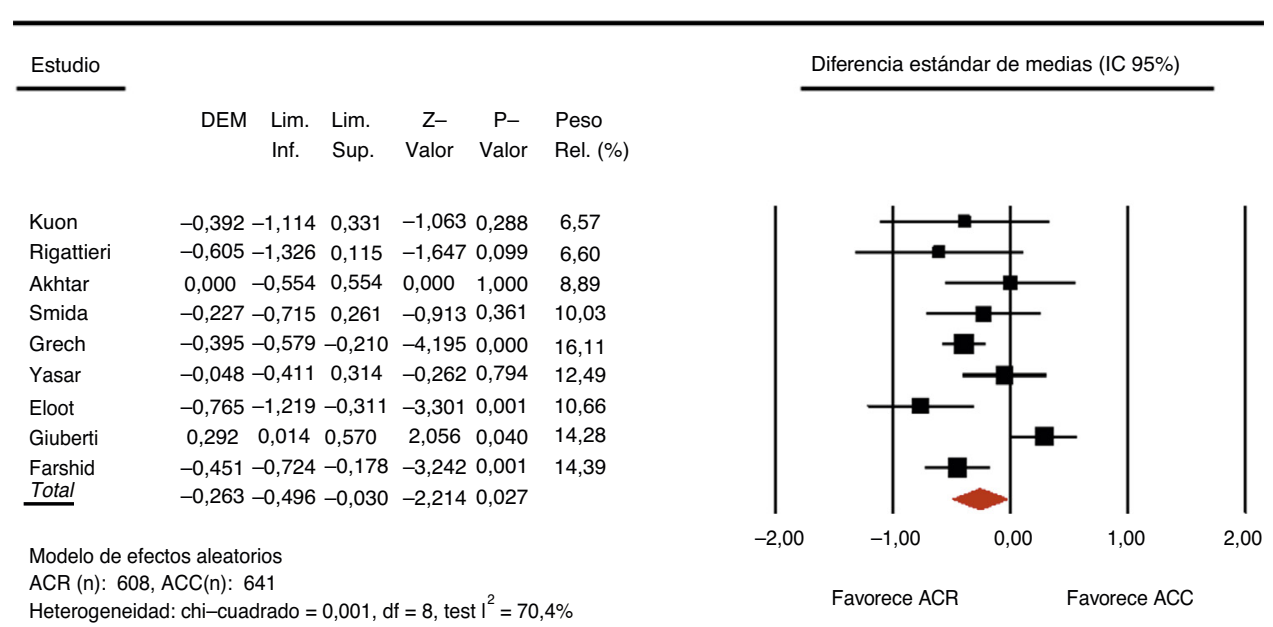

Figura 5 Diagrama de bosque comparando el tiempo de fluoroscopia (s) entre la angiografía coronaria rotacional (ACR) y la angiografía coronaria convencional (ACC) (IC 95\%: Intervalo de confianza del 95\%; DEM: diferencia estándar de medias; IC 95\%: Intervalo de confianza del 95\%; Inf.: inferior; Lim.: límite; Rel.: relativo; Sup.: superior.

\section{Conclusiones}

La realización de la ACR, en contraposición a la ACC, permite reducir el volumen de contraste administrado y la exposición a las radiaciones ionizantes en pacientes a los que se les realizan procedimientos coronarios invasivos.

\section{Responsabilidades éticas}

Protección de personas y animales. Los autores declaran que para esta investigación no se han realizado experimentos en seres humanos ni en animales.
Confidencialidad de los datos. Los autores declaran que han seguido los protocolos de su centro de trabajo sobre la publicación de datos de pacientes.

Derecho a la privacidad y consentimiento informado. Los autores declaran que en este artículo no aparecen datos de pacientes.

\section{Financiación}

No se recibió patrocinio de ningún tipo para llevar a cabo este artículo. 


\section{Conflicto de intereses}

\section{Los autores declaran no tener ningún conflicto de intereses.}

\section{Bibliografía}

1. McCullough PA, Wolyn R, Rocher LL, et al. Acute renal failure after coronary intervention: Incidence, risk factors, and relationship to mortality. Am J Med. 1997;103:368.

2. Rihal CS, Textor SC, Grill DE, et al. Incidence and prognostic importance of acute renal failure after percutaneous coronary intervention. Circulation. 2002;105:2259-64.

3. Karatzis EN, Danias PG. Exposure to ionizing radiation from cardiovascular imaging and therapeutic procedures may be a considerable unrecognized risk for subsequent cancer development. J Am Coll Radiol. 2008;5:694-5.

4. Topol EJ, Nissen SE. Our preoccupation with coronary luminology. The dissociation between clinical and angiographic findings in ischemic heart disease. Circulation. 1995;92:2333-42.

5. Galbraith JE, Murphy ML, de Soyza N. Coronary angiogram interpretation. Interobserver variability. JAMA. 1978;240: 2053-6.

6. Tommasini G, Camerini A, Gatti A, et al. Panoramic coronary angiography. J Am Coll Cardiol. 1998;31:871-7.

7. Hudson PA, Klein AJ, Kim MS, et al. A novel dual-axis rotational coronary angiography evaluation of coronary artery disease-Case presentation and review. Clin Cardiol. 2010;33: E16-9.

8. Loomba RS, Rios R, Buelow M, et al. Comparison of contrast volume, radiation dose, fluoroscopy time, and procedure time in previously published studies of rotational versus conventional coronary angiography. Am J Cardiol. 2015;116:43-9.

9. Jüni $P$, Altman DG, Egger $M$. Systematic reviews in health care: Assessing the quality of controlled clinical trials. BMJ. 2001;323:42-6.

10. Downs SH, Black N. The feasibility of creating a checklist for the assessment of the methodological quality both of randomised and non-randomised studies of health care interventions. $J$ Epidemiol Community Health. 1998;52:377-84.

11. Kuon E, Niederst PN, Dahm JB. Usefulness of rotational spin for coronary angiography in patients with advanced renal insufficiency. Am J Cardiol. 2002;90:369-73.

12. Maddux JT, Wink O, Messenger JC, et al. Randomized study of the safety and clinical utility of rotational angiography versus standard angiography in the diagnosis of coronary artery disease. Catheter Cardiovasc Interv. 2004;62:167-74.

13. Rigattieri S, Ghini AS, Silvestri P, et al. A randomized comparison between rotational and standard coronary angiography. Minerva Cardioangiol. 2005;53:1-6.

14. Akhtar M, Vakharia KT, Mishell J, et al. Randomized study of the safety and clinical utility of rotational vs. standard coronary angiography using a flat-panel detector. Catheter Cardiovasc Interv. 2005;66:43-9.

15. Smida W, Sidéris G, Stratiev V, et al. Rotational coronary angiography. Arch Mal Coeur Vaiss. 2007;100:895-900.

16. Garcia JA, Agostoni P, Green NE, et al. Rotational vs. standard coronary angiography: An image content analysis. Catheter Cardiovasc Interv. 2009;73:753-61.
17. Empen K, Kuon E, Hummel A, et al. Comparison of rotational with conventional coronary angiography. Am Heart J. 2010;160:552-63.

18. Klein AJ, Garcia JA, Hudson PA, et al. Safety and efficacy of dual-axis rotational coronary angiography vs. standard coronary angiography. Catheter Cardiovasc Interv. 2011;77:820-7.

19. Gómez-Menchero AE, Díaz JF, Sánchez-González C, et al. Comparison of dual-axis rotational coronary angiography (XPERSWING) versus conventional technique in routine practice. Rev Esp Cardiol (Engl Ed). 2012;65:434-9.

20. Liu HL, Jin ZG, Yang SL, et al. Randomized study on the safety and efficacy of dual-axis rotational versus standard coronary angiography. Chin Med J (Engl). 2012;125:1016-22.

21. Grech M, Debono J, Xuereb RG, et al. A comparison between dual axis rotational coronary angiography and conventional coronary angiography. Catheter Cardiovasc Interv. 2012;80:576-80.

22. Yasar AS, Perino AC, Dattilo PB, et al. Comparison of a safety strategy using transradial access and dual-axis rotational coronary angiography with transfemoral access and standard coronary angiography. J Interv Cardiol. 2013;26:524-9.

23. Eloot L, Bacher K, Steenbeke F, et al. Three-dimensional rotational X-ray acquisition technique is reducing patients' cancer risk in coronary angiography. Catheter Cardiovasc Interv. 2013;82:E419-27.

24. Giuberti RS, Caixeta A, Carvalho AC, et al. A randomized trial comparing dual axis rotational versus conventional coronary angiography in a population with a high prevalence of coronary artery disease. J Interv Cardiol. 2014;27:456-64.

25. Farshid A, Chandrasekhar J, McLean D. Benefits of dual-axis rotational coronary angiography in routine clinical practice. Heart Vessels. 2014;29:199-205.

26. Morris PD, Taylor J, Boutong S, et al. When is rotational angiography superior to conventional single-plane angiography for planning coronary angioplasty? Catheter Cardiovasc Interv. 2016;87:E104-12.

27. Hou SH, Bushinsky DA, Wish JB, et al. Hospital acquired renal insufficiency: A prospective study. Am J Med. 1983;74:243-8.

28. Raposeiras-Roubín S, Abu-Assi E, Ocaranza-Sánchez R, et al. Dosing of iodinated contrast volume: A new simple algorithm to stratify the risk of contrast-induced nephropathy in patients with acute coronary syndrome. Catheter Cardiovasc Interv. 2013;82:888-97.

29. Faulkner K, Werduch A. An estimate of the collective dose to the European population from cardiac $\mathrm{X}$-ray procedures. $\mathrm{Br} \mathrm{J}$ Radiol. 2008;81:955-62.

30. Chen J, Einstein A, Fazel R, et al. Cumulative exposure to ionizing radiation from diagnostic and therapeutic cardiac imaging procedures. J Am Coll Cardiol. 2010;56:702-11.

31. Chida K, Kagaya K, Saito H, et al. Evaluation of patient radiation dose during cardiac interventional procedures: What is the most effective method? Acta Radiol. 2009;50:474-81.

32. Bogaert E, Bacher K, Lemmens K, et al. A large-scale multicentre study of patient skin doses in interventional cardiology: Dose-area product action levels and dose reference levels. $\mathrm{Br} \mathrm{J}$ Radiol. 2009;82:303-12.

33. Padovani R, Vano E, Trianni A, et al. Reference levels at European level for cardiac interventional procedures. Radiat Prot Dosimetry. 2008;129:104-7. 